\title{
Comparing microvascular alterations during minimal extracorporeal circulation and conventional cardiopulmonary bypass in coronary artery bypass graft surgery: A prospective, randomized study
}

\author{
Peter Donndorf, MD, ${ }^{a}$ Franziska Kühn, MD, ${ }^{a}$ Brigitte Vollmar, MD, PhD, ${ }^{b}$ Jan Rösner, MD, PhD, ${ }^{c}$ \\ Andreas Liebold, MD, PhD, ${ }^{\mathrm{d}}$ Philipp Gierer, $\mathrm{MD}, \mathrm{PhD},{ }^{\mathrm{e}}$ Gustav Steinhoff, $\mathrm{MD}, \mathrm{PhD},{ }^{\mathrm{a}}$ and \\ Alexander Kaminski, MD, $\mathrm{PhD}^{\mathrm{a}}$
}

\begin{abstract}
Objectives: Minimal extracorporeal circulation (MECC) has been introduced in coronary artery bypass graft (CABG) surgery, offering clinical benefits owing to reduced hemodilution and no blood-air interface. Yet, the effects of MECC on the intraoperative microvascular perfusion in comparison with conventional extracorporeal circulation (CECC) have not been studied so far.
\end{abstract}

\begin{abstract}
Methods: The current study aimed to analyze alterations in microvascular perfusion at 4 predefined time points (T1-T4) during on-pump CABG using orthogonal polarization spectral imaging. Forty patients were randomized for being operated on with either MECC or CECC. Changes in functional capillary density (FCD), blood flow velocity, and vessel diameter were analyzed by a blinded investigator.
\end{abstract}

\begin{abstract}
Results: After start of extracorporeal circulation (ECC) and aortic crossclamping (T2), both groups showed a significant drop of FCD, with a significantly higher FCD in the MECC group $\left(206.8 \pm 33.6 \mathrm{~cm} / \mathrm{cm}^{2}\right.$ in CECC group versus $217.8 \pm 35.3 \mathrm{~cm} / \mathrm{cm}^{2}$ in MECC group; $P=.034$ ). In the late phase of the ECC (T3), FCD in the MECC group was already recovered, whereas FCD in the CECC group was still significantly depressed $\left(223.1 \pm 35.6 \mathrm{~cm} / \mathrm{cm}^{2}\right.$ in MECC group; $P=.100$ vs T1; $211.1 \pm 36.9 \mathrm{~cm} / \mathrm{cm}^{2}$ in CECC group; $P=.017$ vs T1). After termination of ECC (T4), FCD recovered in both groups to baseline. Blood flow velocity tended to be higher in the MECC group, with a significant intergroup difference after aortic crossclamping (T2).

Conclusions: Orthogonal polarization spectral imaging data reveal an impairment of microvascular perfusion during on-pump CABG. Changes in FCD indicate a faster recovery of the microvascular perfusion in MECC during the reperfusion period. Beneficial recovery of microvascular organ perfusion could partly explain the perioperative advantages reported for MECC. (J Thorac Cardiovasc Surg 2012;144:677-83)
\end{abstract}

\begin{abstract}
Although the rate of complications of coronary artery bypass graft $(\mathrm{CABG})$ surgery with cardiopulmonary bypass (CPB) has constantly decreased during the past 3 decades, postoperative neurologic and neurobehavioral disorders, ${ }^{1}$ fulfilling the definition of delirium in $6 \%$ of the cases, ${ }^{2}$ as well as a certain degree of myocardial damage are still common. These complications and side effects are primarily considered to be caused by gaseous and solid microemboli $^{3,4}$ as well as by ischemia-reperfusion injury. However, changes particularly in microvascular perfusion might also play a relevant role in causing these complications owing
\end{abstract}

\footnotetext{
From the Department of Cardiac Surgery, ${ }^{\mathrm{a}}$ Institute of Experimental Surgery, ${ }^{\mathrm{b}}$ and Department of Anesthesiology and Intensive Care Medicine, ${ }^{c}$ University of Rostock, Rostock, Germany; the Department of Cardiovascular and Thoracic Surgery, ${ }^{\mathrm{d}}$ University of Ulm, Ulm, Germany; and the Department of Trauma and Reconstructive Surgery, ${ }^{\mathrm{e}}$ University of Rostock, Rostock, Germany.

Disclosures: Authors have nothing to disclose with regard to commercial support.

Received for publication Nov 16, 2011 ; revisions received April 4, 2012; accepted for publication May 15, 2012; available ahead of print June 14, 2012.

Address for reprints: Peter Donndorf, MD, Department of Cardiac Surgery, University of Rostock, Schillingallee 35, 18057 Rostock, Germany, Phone/Fax: +49-381494-6101/-6102 (E-mail: peter.donndorf@med.uni-rostock.de). $0022-5223 / \$ 36.00$

Copyright (c) 2012 by The American Association for Thoracic Surgery doi:10.1016/j.jtcvs.2012.05.037
}

to the known relevance of microcirculation in other perioperative organ dysfunctions. ${ }^{5,6}$ Inasmuch as these side effects can be attributed in a large part to CPB, continuous efforts have been made to reduce these effects. Consequently, besides establishment of off-pump procedures with complete avoidance of CPB, minimal extracorporeal circulation (MECC) systems have been developed and introduced successfully in the clinical routine. ${ }^{7,8}$ The concept is based on the idea of a closed circuit with a low prime volume consisting of a centrifugal blood pump and a modern low-volume membrane oxygenator as the only components. The venous blood returns through active drainage. Neither a venous reservoir nor a cardiotomy suction device is used. The shed blood is separated from the systemic circulation. The components, including the tubing, are heparin coated. In this way the MECC system offers possible clinical benefits owing to reduced hemodilution, no blood-air interface, and consecutively attenuated inflammatory response. MECC might be especially useful in the growing cohort of patients being referred for surgery in advanced stages of coronary disease, requiring complex multivessel grafting, by offering a surgical setting in which completeness of revascularization is warranted 


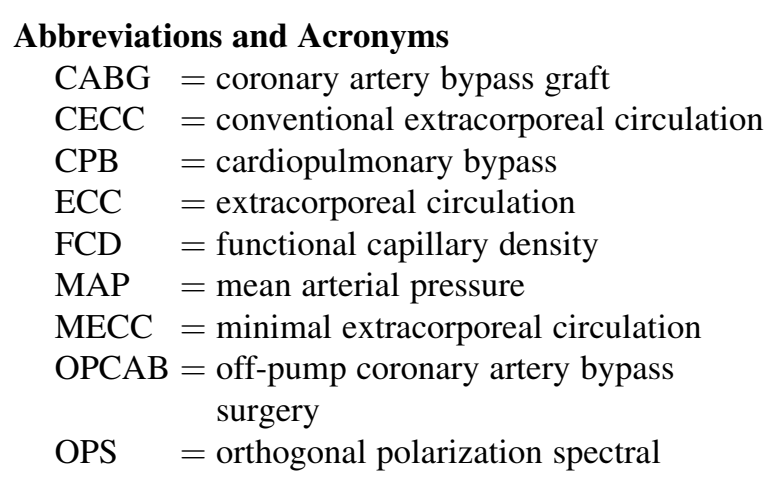

and high-risk patients can be operated on safely. ${ }^{9}$ Therefore, with every surgeon feeling confident using this system, MECC already forms a standard procedure during CABG at our institution as a tool for preservation of lower hemodilution as the most obvious clinical parameter. However, the effects of MECC systems on the intraoperative microvascular perfusion in comparison with conventional extracorporeal circulation (CECC) have not been studied conclusively so far. With the aim to evaluate possible beneficial effects of the MECC system on microcirculatory alterations during on-pump CABG, we conducted a prospective randomized study comparing the MECC system with conventional CPB regarding changes in microvascular perfusion of the sublingual mucosa. For microcirculatory analyses, orthogonal polarization spectral (OPS) imaging, a noninvasive visualization system of microvascular perfusion, was used. ${ }^{10}$ Inasmuch as the nutritive blood flow of the sublingual mucosa is known to correlate with that of vital organs, ${ }^{11,12}$ it is currently the most often used site for OPS measurements in humans,${ }^{10}$ and data on its alterations provide relevant clinical information about the microvascular perfusion during on-pump cardiac surgery.

\section{METHODS}

\section{Patient Selection}

Forty patients scheduled for urgent or elective CABG surgery were enrolled between June and November 2010. Exclusion criteria were carotid artery stenosis greater than $60 \%$, age older than 80 years, atrial fibrillation, left ventricular ejection fraction of less than $30 \%$, emergency operation, reoperation, or combined valve surgery. Patients were randomized into 2 groups (CECC and MECC groups) by computer-generated random allocations. The study was approved by the institutional ethics committee and all patients provided written informed consent.

\section{Perfusion Technology}

Both the CECC and the MECC, as used in the current study, are used routinely at our institution in CABG cases. Either system features complete heparin coating (Bioline Coating; Maquet Cardiopulmonary, Rastatt, Germany) of the tubing system. Detailed specifications on the components of the 2 perfusion circuits are given in Table 1.

Anticoagulation in the CECC group was attained by administration of $300 \mathrm{IU} / \mathrm{kg}$ of heparin to achieve an activated clotting time of more than 400 seconds. In the MECC group, $200 \mathrm{IU} / \mathrm{kg}$ of heparin was administered with a target activated clotting time of 250 seconds. For both groups, alpha-stat blood gas management was applied. The extracorporeal flow rate for both systems was set at $2.4 \mathrm{~L} \cdot \mathrm{min}^{-1} \cdot \mathrm{m}^{-2}$.

\section{Anesthesia}

A standardized anesthetic protocol was used for all patients. Anesthesia was induced with sufentanil $\left(0.25 \mu \mathrm{g} \cdot \mathrm{kg}^{-1}\right.$; Janssen-Cilag $\mathrm{GmbH}$, Neuss, Germany) and etomidate $\left(0.2 \mathrm{mg} \cdot \mathrm{kg}^{-1} ; \mathrm{B}\right.$. Braun Melsungen AG, Melsungen, Germany). After relaxation with rocuronium $\left(0.6 \mathrm{mg} \cdot \mathrm{kg}^{-1}\right.$; ORGANON GmbH, Oberschleißheim, Germany), patients were orally intubated with an orotracheal tube (Covidien, Mansfield, Mass). Anesthesia was maintained with continuous infusion of propofol $\left(4 \mathrm{mg} \cdot \mathrm{kg}^{-1} \cdot \mathrm{h}^{-1} ; \mathrm{B}\right.$. Braun Melsungen AG), sufentanil $\left(1 \mu \mathrm{g} \cdot \mathrm{kg}^{-1}\right.$; Janssen-Cilag $\mathrm{GmbH}$, Neuss, Germany), and cisatracurium (Glaxo Smithkline $\mathrm{GmbH} \& \mathrm{Co}$ KG, Munich, Germany). Pressure-controlled mechanical ventilation was provided by a Primus anesthesia ventilator (Draeger, Luebeck, Germany). Respiratory rate and pressure were adjusted to maintain an arterial partial pressure of carbon dioxide at 4.8 to $5.6 \mathrm{kPa}$. A positive end-expiratory airway pressure of up to $5 \mathrm{~cm} \mathrm{H}_{2} \mathrm{O}$ was applied to maintain an arterial partial pressure of oxygen at 12 to $15 \mathrm{kPa}$.

\section{Surgical Intervention and Cardioplegia}

After median sternotomy, the left internal thoracic artery and additional venous graft material were harvested. Heparinization was followed by standard venous and arterial cannulation: a $24 \mathrm{~F}$ arterial cannula for the ascending aorta and a $32 \mathrm{~F} / 37 \mathrm{~F} 2$-stage cannula for the right atrial appendage. Venting was accomplished by using a needle in the ascending aorta, which was connected through a drop chamber to the venous line (MECC group) or to the reservoir (CECC group). Cardioplegic solution (Calafiore, a mixture of arterial blood, $10 \mathrm{~mL}$ of $\mathrm{KCl} 14.9 \%$, and $2 \mathrm{~mL}$ of $\mathrm{MgSO}_{4} 50 \mathrm{vol} / \mathrm{mL}$ ) was administered through the aortic root after aortic crossclamping. Surgical intervention was performed during mild hypothermia (esophageal temperature, $33^{\circ} \mathrm{C}-34^{\circ} \mathrm{C}$ ). The coronary anastomoses were constructed by hand suturing. Boluses of cardioplegic solution were given in 20-minute intervals. Proximal anastomoses were done on the side-clamped aorta during reperfusion. After rewarming, the patient was weaned off $\mathrm{CPB}$, and heparin was neutralized.

\section{OPS Imaging}

The imaging technique has been described in detail previously. ${ }^{13,14}$ In brief, sublingual microcirculation was observed using the Cytoscan System (Cytoscan; Cytometrics Incorporated, Philadelphia, Pa), consisting of an optical probe connected to an external light source via liquid light guide cable. With the use of a standard video recorder (S-VHS, AG 7350-E, Panasonic; Matsushita Electric Ind, Osaka, Japan) and a standard video screen, online imaging as well as the recording of obtained images for later offline analysis were performed. On the video screen, a final 465-fold magnification was achieved. Erythrocytes were visualized by the passage of light through a spectral filter to isolate the wavelength of $548 \mathrm{~nm}$ (isobestic point of hemoglobin). The polarized light was then emitted to the target by a beam splitter. The light, sent back from the target, was collected by the same lens. All images obtained of the illuminated region were captured using a charge-coupled device video camera.

Similar to the epi-illumination intravital microscopy, OPS imaging allows the measurement of the vessel diameter, the length of the perfused vessels per observation area, and the flow velocity of the red blood cells inside the vessel.

OPS images were obtained from the sublingual mucosa where the probe was put in direct contact with the tissue. Once blood vessels were identified, the probe was manually focused. By stabilizing the probe gently on the patient's teeth, it was possible to obtain good images with minimal movement artifacts. Care was taken to minimize the contact pressure 
TABLE 1. Major components of the perfusion circuits used

\begin{tabular}{lll}
\hline & \multicolumn{1}{c}{ CECC } & \multicolumn{1}{c}{ MECC } \\
\hline Priming volume $(\mathrm{mL})$ & $1500 \mathrm{~mL}$ crystalloid/colloid & $800 \mathrm{~mL}$ crystalloid \\
Venous reservoir & Yes & No \\
Membrane oxygenator & Quadrox-i* & Quadrox D* \\
Cardiotomy suction & Yes & No \\
Arterial line filter & Yes & No \\
Pump & Roller pump & Centrifugal pump \\
\hline
\end{tabular}

$C E C C$, Conventional extracorporeal circulation; $M E C C$, minimal extracorporeal circulation. *Maquet Cardiovascular, Wayne, New Jersey.

necessary for the venules and capillaries to remain in focus. In this way artificial impairment of the sublingual blood flow could be minimized. OPS images were recorded at 4 time points: after induction of anesthesia before skin incision (T1, baseline), after the start of CPB and aortic crossclamping (T2), 10 minutes after release of the aortic crossclamp (T3), and 30 minutes after termination of CPB (T4) (Figure 1). At each time point 4 to 5 sequences of 30 seconds were recorded. All visible microvessels in each sequence were analyzed. For the quantitative offline analysis, a computer-assisted microcirculation image-analysis system was used (CapImage version 7.4; Zeintl, Heidelberg, Germany). Offline analysis included red blood cell velocity, vessel diameter, as well as functional capillary density (FCD), which is the total length of red blood cell-perfused capillaries per observation area, given in centimeters per square centimeters, and was performed by a blinded investigator.

\section{Hemodynamic Monitoring}

Blood pressure (systolic, diastolic, and mean), heart rate, arterial oxygen saturation, $\mathrm{PO}_{2} / \mathrm{PCO}_{2}$, central venous oxygen saturation, arterial lactate, as well as hemoglobin and hematocrit were monitored and recorded at the 4 time points of microvascular measurements described earlier.

\section{Study Conditions and Recording of Clinical Parameters}

Several study conditions were predefined for standardization. The operations were performed by 4 experienced surgeons. The random allocation of the perfusion method was not communicated to the surgeon before the operation. During perfusion, hematocrit values were not allowed to decrease to less than 0.25; otherwise, packed red cells were transfused. When mean arterial pressure (MAP) decreased to less than $45 \mathrm{~mm} \mathrm{Hg}$, a bolus of $0.01 \mathrm{mg}$ of norepinephrine was administered. Units of packed red cells transfused, norepinephrine use, and intraoperative clinical events were recorded. The study parameters were entered into a computer database and processed in a blinded manner by a single investigator.

\section{Sample Size Calculation and Statistical Analysis}

The primary outcome variable was the change in FCD. A sample size of 40 patients ( 20 per group) provided $80 \%$ power $(\alpha=.05, \beta=.2)$ to detect an absolute difference in change of FCD of $5 \%$ between the groups by using 2-way analysis of variance (version 5.0, nQuery Advisor; Statistical Solutions Ltd, Cork, Ireland). We randomized 40 patients by using a 1:1 randomization (20 patients per group). Continuous data are expressed as means \pm standard error of mean. Changes in FCD, vessel diameter, red blood cell velocity, hematocrit, and lactate over time were analyzed by repeated-measures analysis of variance and pairwise multiple comparison procedure (Holm-Sidak method). Intergroup comparison for preoperative, perioperative, and postoperative data was assessed by the Student $t$ test for continuous variables and the Fisher exact test for proportions. For correlation analysis, Pearson product moment correlation was applied. Statistical analysis was performed with the SPSS software package (version 12.0; SPSS Inc, Chicago, Ill).

\section{RESULTS}

All patients completed the study. Patient demographic data and baseline intraoperative characteristics are shown
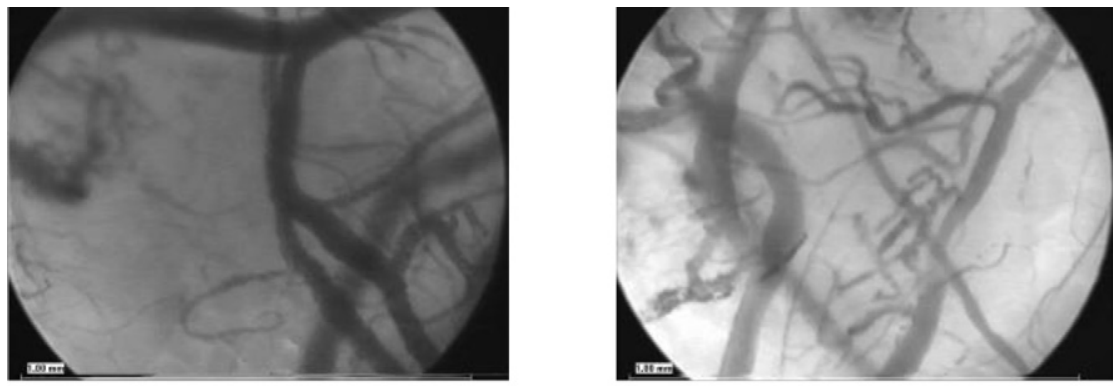

T1

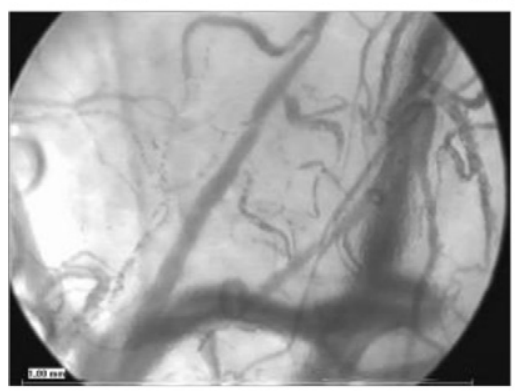

T3
OPS- Imaging

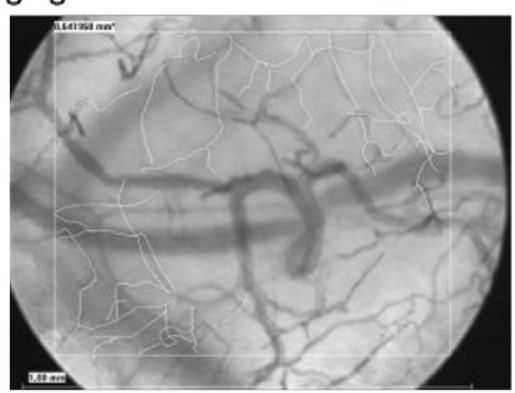

T4

FIGURE 1. Representative images of sublingual microcirculation obtained by orthogonal polariztion spectral (OPS) imaging at 4 time points during on-pump coronary artery bypass grafting: $T 1$, at skin incision (baseline); $T 2,10$ minutes after aortic crossclamping; $T 3,10$ minutes after release of the aortic crossclamp; T4, 30 minutes after termination of cardiopulmonary bypass (manual offline measurement of the sublingual functional capillary density by line drawing is illustrated). 
TABLE 2. Patient characteristics and intraoperative data

\begin{tabular}{lccc}
\hline \multicolumn{1}{c}{ Variable } & $\begin{array}{c}\text { CECC group } \\
(\mathbf{n}=\mathbf{2 0})\end{array}$ & $\begin{array}{c}\text { MECC group } \\
(\mathbf{n}=\mathbf{2 0})\end{array}$ & $\begin{array}{c}\boldsymbol{P} \\
\text { value }\end{array}$ \\
\hline Age (y) & $69 \pm 8$ & $67 \pm 8$ & .57 \\
Male sex (\%) & 80 & 70 & .71 \\
Diabetes (\%) & 55 & 45 & .75 \\
pVD (\%) & 20 & 20 & 1.0 \\
Nicotin (\%) & 35 & 55 & .34 \\
EuroSCORE (\%) & $4.0 \pm 3.9$ & $3.4 \pm 2.0$ & .50 \\
Crossclamp time (min) & $56 \pm 18$ & $54 \pm 16$ & .78 \\
Total CPB time (min) & $97 \pm 24$ & $96 \pm 27$ & .84 \\
Intraoperative vasopressors (\%) & 65 & 55 & .75 \\
\hline Data are presented as means \pm standard error of the mean where shown. $C E C C$, Con- \\
ventional extracorporeal circulation; MECC, minimized extracorporeal circulation; \\
pVD, peripheral vascular disease; EuroSCORE, European system for cardiac opera- \\
tive risk evaluation; $C P B$, cardiopulmonary bypass.
\end{tabular}

in Table 2. There were no significant differences between the study groups regarding age, gender distribution, presence of diabetes, nicotine abuse, peripheral vascular disease, crossclamp time, total CPB time, or the need for intraoperative norepinephrine use (Table 2). No postoperative myocardial infarctions or early major organ complications occurred. All patients except one were discharged from the hospital in stable cardiopulmonary condition. One patient (CECC group) died on the eighth postoperative day of acute dissection of a pre-existing infrarenal aortic aneurysm.

\section{Intraoperative Hemodynamics, Lactate Level, and Hematocrit Level}

The baseline MAP did not differ significantly between the groups $(72.2 \pm 14.0 \mathrm{~mm} \mathrm{Hg}$ in CECC group vs $70.3 \pm 9.8$ in MECC group at T1; $P=.174$ ). There was a significant difference between the groups regarding MAP at the time points T2 $(50.0 \pm 10.8 \mathrm{~mm} \mathrm{Hg}$ in CECC group vs $60.9 \pm 15.9$ in MECC group; $P=.013$ ) and T4 $(72.6 \pm 8.8 \mathrm{~mm} \mathrm{Hg}$ in CECC group vs $80.5 \pm 11.1 \mathrm{~mm}$ $\mathrm{Hg}$ in MECC group; $P=.014$ ) with a higher MAP in the MECC group, but not at T3 $(52.5 \pm 7.4$ in CECC group vs $54.1 \pm 9.6 \mathrm{~mm} \mathrm{Hg}$ in MECC group; $P=.431$ ) (Figure 2). The intraoperative changes in serum lactate levels did not differ significantly between the groups (Figure 3). Regarding intraoperative hematocrit values, a significant difference between the MECC and the CECC groups was detected at the time points $\mathrm{T} 2(27.7 \pm 4.0$ in CECC group vs $33.5 \pm 4.8$ in MECC group; $P<.001$ ) and T3 (30.1 \pm 11.0 in CECC group vs $33.1 \pm 5.0$ in MECC group; $P=.011$ ), indicating a more marked hemodilution in the CECC group during CPB (Figure 3).

\section{Microvascular Alterations Measured by OPS Imaging}

In general, the OPS imaging technique proved feasible in terms of visualizing sublingual microcirculation during

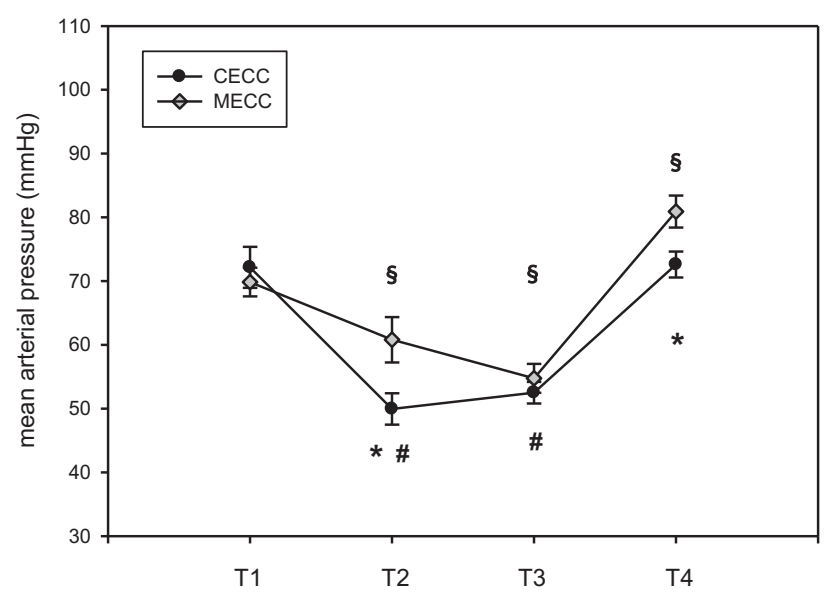

FIGURE 2. Intraoperative mean arterial pressure ( $\mathrm{mm} \mathrm{Hg}$ ) during coronary artery bypass graft surgery measured at 4 time points: $T 1$, after skin incision (baseline); T2, 10 minutes after aortic crossclamping; T3, 10 minutes after release of the aortic crossclamp; T4, 30 minutes after termination of cardiopulmonary bypass. The line graphs illustrate the variation of the mean arterial pressure during on-pump coronary bypass grafting using minimized (MECC, $\mathrm{n}=20$ patients) or conventional (CECC, $\mathrm{n}=20$ patients) extracorporeal circulation. Asterisks denote statistically significant intergroup differences. Symbols indicate significant differences relative to baseline within each group: $* P<.05$, MECC versus CECC; $\# P<.05$ versus T1 CECC (baseline); $\S P<.05$ versus T1 MECC (baseline). Data are presented as means \pm standard error of the mean.

cardiac surgery. Independent from the type of extracorporeal circulation (ECC), a certain degree of impairment regarding the microvascular perfusion during $\mathrm{CPB}$ was detectable.

Functional capillary density. There was no significant difference in the baseline level of the FCD between the 2 groups $\left(224.8 \pm 38.8 \mathrm{~cm} / \mathrm{cm}^{2}\right.$ in CECC group vs 232.6 $\pm 37.3 \mathrm{~cm} / \mathrm{cm}^{2}$ in MECC group; $\left.P=.246\right)$. After the start of the ECC and aortic crossclamping (T2), both groups showed a significant drop of the FCD but with a significantly higher FCD in patients operated on with the MECC system $\left(206.8 \pm 33.6 \mathrm{~cm} / \mathrm{cm}^{2}\right.$ in CECC group vs $217.8 \pm 35.3 \mathrm{~cm} /$ $\mathrm{cm}^{2}$ in MECC group; $\left.P=.034\right)$. At T3, in the late phase of $\mathrm{CPB}$, the FCD in the MECC group was still impaired but not significantly below the baseline value, whereas in the CECC group it was still significantly depressed $(223.1 \pm 35.6 \mathrm{~cm} /$ $\mathrm{cm}^{2}$ in MECC group; $P=.100$; vs T1; $211.1 \pm 36.9 \mathrm{~cm} / \mathrm{cm}^{2}$ in CECC group; $P=.017$ vs T1). Intergroup comparison, however, did not reveal a significant difference of the FCD between the 2 groups at the time point T3 $(P=.071)$. After termination of $\mathrm{CPB}$, the FCD recovered in both groups to baseline values (Figure 4). There was no significant correlation detectable between the FCD and the respective MAP in the CECC group (correlation coefficient $=0.275$; $P=.104)$. The MECC group showed a weak but significant positive correlation between these 2 parameters (correlation coefficient $=0.357 ; P=.023$ ). 

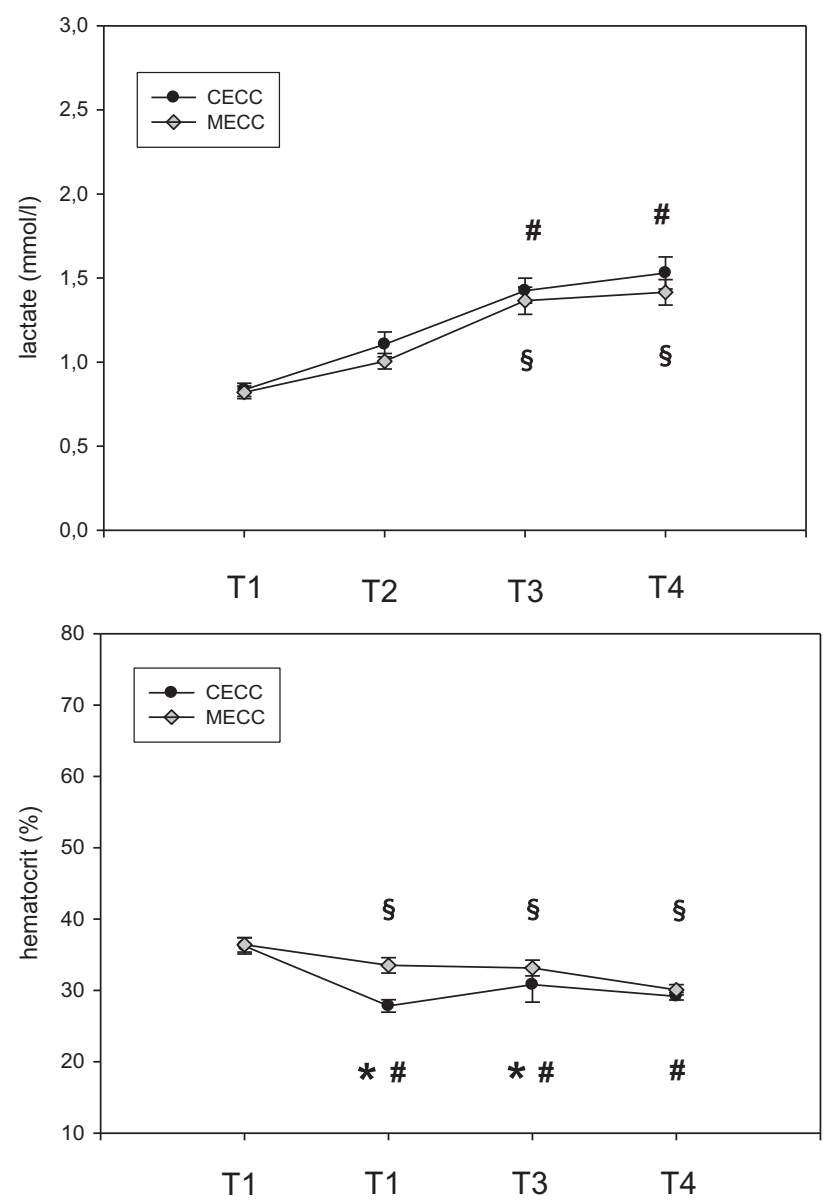

FIGURE 3. Intraoperative serum lactate $(\mathrm{mmol} / \mathrm{L})$ and hematocrit $(\%)$ during coronary artery bypass graft surgery measured at 4 time points: $T 1$, after skin incision (baseline); $T 2,10$ minutes after aortic crossclamping; T3, 10 minutes after release of the aortic crossclamp; T4, 30 minutes after termination of the $\mathrm{CPB}$. The line graphs illustrate the variation of the serum lactate level (upper panel) and the hematocrit (lower panel) during on-pump coronary bypass grafting using minimized (MECC, $\mathrm{n}=20$ patients) or conventional (CECC, $\mathrm{n}=20$ patients) extracorporeal circulation. Asterisks denote statistically significant intergroup differences. Symbols indicate significant differences relative to baseline within each group. $* P<.05$ MECC versus CECC; $\# P<.05$ versus T1 CECC (baseline); $\S P<.05$ versus T1 MECC (baseline). Data are presented as means \pm standard error of the mean.

Vessel diameter and blood flow velocity. There was no significant difference detectable between the groups regarding the diameters of postcapillary venules in the sublingual microcirculation measured by OPS at the 4 respective time points (data not shown). Measurements of red blood cell velocity in postcapillary venules revealed no significant intraoperative changes during $\mathrm{CABG}$ operations using ether CECC or MECC. However, the blood flow velocity tended to be higher in the MECC group, with a significant intergroup difference at the time point $\mathrm{T} 2(0.510 \pm 0.014 \mathrm{~mm} / \mathrm{s}$ in CECC group vs $0.553 \pm 0.012 \mathrm{~mm} / \mathrm{s}$ in MECC group; $P=.011$ ) (Figure 5).

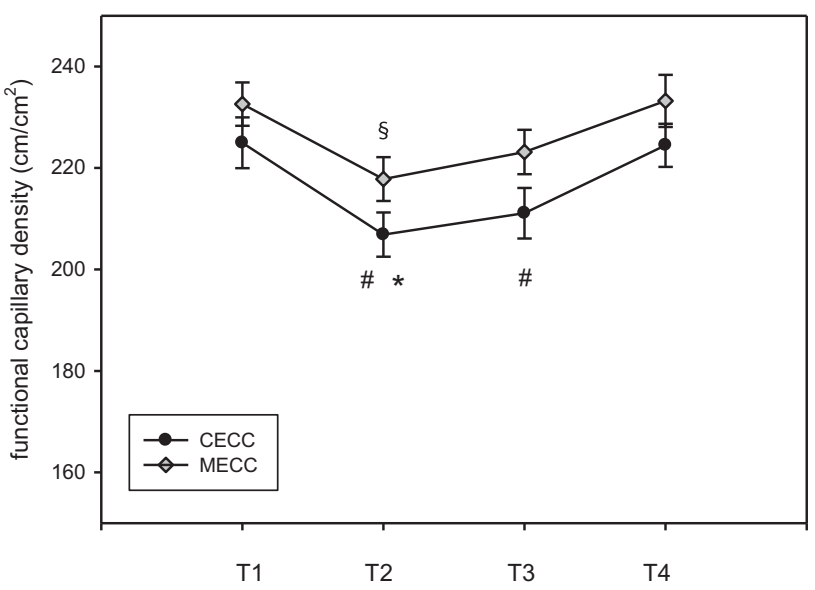

FIGURE 4. Functional capillary density $(F C D)$ of the sublingual microcirculation measured by OPS imaging at 4 time points: $T 1$, after skin incision (baseline); T2, 10 minutes after aortic crossclamping; T3, 10 minutes after release of the aortic crossclamp; T4, 30 minutes after termination of cardiopulmonary bypass. The line graphs illustrate the variation of the FCD during on-pump coronary bypass grafting using conventional (CECC, $\mathrm{n}=20$ patients) and minimal (MECC, $\mathrm{n}=20$ patients) extracorporeal circulation. Asterisks denote statistically significant intergroup differences. Symbols indicate significant differences relative to baseline within each group. ${ }^{*} P<.05$ MECC versus CECC; $\# P<.05$ versus T1 CECC (baseline); $\S P<.05$ versus T1 MECC (baseline). Data are presented as means \pm standard error of the mean.

\section{DISCUSSION}

MECC systems have been used for several years in CABG surgery to reduce side effects associated with ECC. Although until today a clear advantage regarding postoperative morbidity and mortality of MECC systems in comparison with CECC has not been conclusively proven in large-scale randomized trials, several prospective studies were able to show beneficial perioperative effects of the MECC system, that is, in terms of postoperative troponin levels, intraoperative cerebral oxygenation, and need for red blood cell transfusions. ${ }^{3,15,16}$ Furthermore, superior early neurocognitive outcome has been recently reported by Anastasiadis and colleagues. ${ }^{17}$ In fact, several of these beneficial perioperative effects are similar to those reported for off-pump coronary artery bypass surgery (OPCAB), ${ }^{18}$ and especially the growing cohort of patients with complex, multivessel lesions and relevant comorbidities might profit from MECC rather than OPCAB in certain situations. However, the intraoperative pathophysiologic mechanisms - possibly resulting from reduced hemodilution and attenuated inflammatory response during MECC — contributing to reported differential outcome of MECC compared with CECC have not been studied conclusively so far. Especially, the reported differences regarding myocardial damage have not been well explained, inasmuch as crossclamp time and cardioplegia protocols are independent from the type of ECC used. Owing the known relevance of 


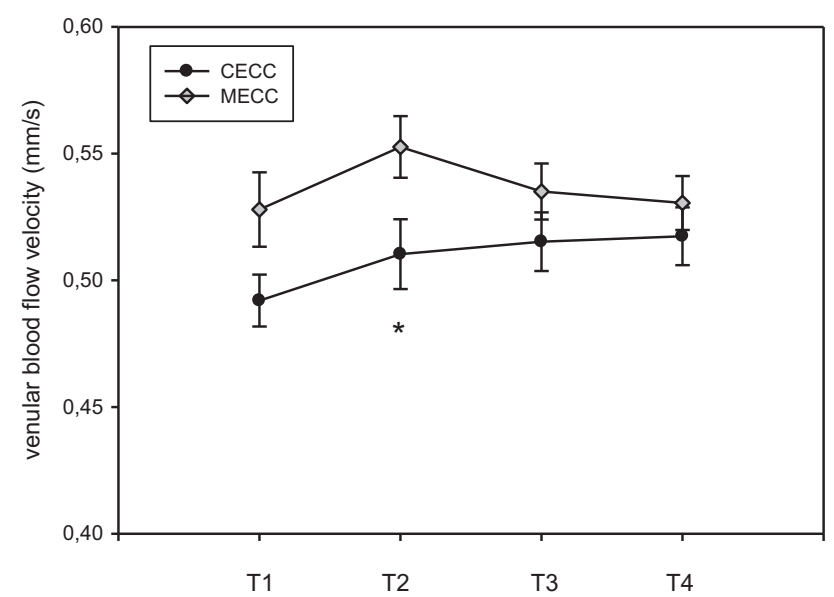

FIGURE 5. Venular blood flow velocity $(F C D, \mathrm{~mm} / \mathrm{s})$ of the sublingual microcirculation measured by orthogonal polarization spectral imaging at 4 time points: $T 1$, after skin incision (baseline); $T 2,10$ minutes after aortic crossclamping; $T 3,10$ minutes after release of the aortic crossclamp; $T 4$, 30 minutes after termination of cardiopulmonary bypass. The line graphs illustrate the variation of the FCD during on-pump bypass grafting using conventional (CECC, $\mathrm{n}=20$ patients) and minimal $(M E C C, \mathrm{n}=20 \mathrm{pa}-$ tients) extracorporeal circulation. Asterisks denote statistically significant intergroup differences. $* P<.05$ MECC versus CECC. Data are presented as means \pm standard error of the mean.

microvascular perfusion on perioperative organ complications, we aimed in this study at comparing microvascular alterations during CABG surgery using either an MECC or a CECC system.

The feasibility of OPS imaging for microvascular blood flow analysis during cardiac surgery has been shown before, as well as the ability of this method also to detect minor changes of microvascular perfusion during CPB. However, to our knowledge, we present here the first study comparing microvascular alterations during MECC and CECC. The sublingual mucosa is particularly useful inasmuch as it offers uncomplicated intraoperative access and its nutritive blood flow, represented by the FCD, is known to correlate with that of vital organs. Like others, we were able to show that, in general, a certain degree of impairment in microvascular perfusion is detectable during $\mathrm{CPB}$ with consecutive nonpulsatile blood flow, hemodilution, and contact activation of the blood. ${ }^{19}$ According to our findings, both MECC and CECC cause alterations in microvascular blood flow. Comparing the MECC system with conventional CPB revealed, however, a faster recovery of sublingual nutritive blood flow in the MECC group, with the FCD being restored close to baseline values in the late phase of CPB. This difference during the reperfusion period might contribute to beneficial perioperative organ protection described for the MECC system, represented by attenuated myocardial damage and better neurologic outcome. ${ }^{15}$ The difference regarding preservation of the microvascular perfusion in the late phase of CPB between the 2 groups could be explained at least partially by the more marked hemodilution in the CECC group compared with the MECC. Preclinical animal models have shown an altered microvascular perfusion during periods of low hematocrit as well as an enhanced neurobehavioral outcome owing to avoidance of hemodilution in selective cerebral perfusion. ${ }^{20,21}$ In line with these findings, Nollert and associates ${ }^{22}$ describe hemodilution as one of the main determinants for impaired cerebral oxygenation during CPB. Although our study lacks a control group with similar surgical conditions without any hemodilution, it is conceivable that reduced hemodilution by a minimized CPB contributes to preservation of the FCD during on-pump CABG. This implication is corroborated by Atasever and colleagues, ${ }^{23}$ reporting no changes in FCD during OPCAB, except for complete cessation of microvascular flow during cardiac luxation maneuvers in OPCAB. Furthermore, reduction of inflammatory stimuli and mechanical stress in MECC systems must be considered as a factor involved in the reported finding. Vice versa, conventional hypothermic CPB is known to be associated with endothelial damage ${ }^{24}$ and consecutive loss of endothelium-dependent regulation of the microcirculatory blood flow. ${ }^{25}$ Microemboli, reported to occur more frequently during MECC than CECC, ${ }^{3,26}$ form another mechanism potentially influencing the intraoperative FCD, as embolic events can cause local microvascular hypoperfusion with a consecutive drop of the FCD. MAPs were not significantly different between the groups at T3, but a weak positive correlation between the FCD and the MAP was detectable in the MECC group. However, the preservation of the FCD is unlikely to be just a pressure-related phenomenon but should be considered as the result of a complex interplay including reduction of hemodilution and embolic events, attenuated inflammatory response, and higher perfusion pressure in the MECC group. Especially, reported moderate benefits in terms of myocardial damage are likely to be influenced by advantageous restoration of nutritive organ perfusion after release of the aortic crossclamp.

Hypothermia represents another factor potentially influencing microvascular perfusion during CPB. However, patients analyzed in the current study were operated on with moderate hypothermia, whereas only deep hypothermia has been shown to be associated with relevant changes in FCD so far. ${ }^{27}$

Interestingly, analysis of red blood cell velocity revealed a tendency of a higher microvascular blood flow velocity in the MECC group, although marked hemodilution, as occurring during CECC, is assumed to contribute to an increase of microvascular blood flow velocity. ${ }^{28}$ The finding is likely to be explained at least partially by the reported difference in systemic perfusion pressure during CPB with higher MAPs in the MECC group, inasmuch as a positive correlation between sublingual blood flow velocity and MAP has been already described by Wiessner and coworkers. ${ }^{29}$ 


\section{CONCLUSIONS}

In conclusion, we were able to show that microcirculatory alterations occur during on-pump CABG independently from the type of perfusion circuit used. However, our data indicate a trend toward faster recovery of microcirculatory blood flow, represented by the FCD, in the MECC group. This suggests superiority of an MECC in terms of restoring nutritive microvascular blood flow. If proven significant in larger patient cohorts, beneficial recovery of microvascular tissue and organ perfusion in MECC systems might represent a mechanism partially contributing to perioperative advantages reported for MECC in CABG surgery.

\section{Limitations}

The problems resulting from movement artifacts as well as contact pressure during OPS imaging have already been discussed extensively. ${ }^{19}$ As concluded by Bauer and colleagues, ${ }^{19}$ we assume that potential error would be random and therefore differences between patients should still be detectable. The MECC system itself is associated with a certain "learning curve," that is, strict avoidance of any air entrance to the circuit and any major bleeding. However, in our experience this step can be easily overcome for CABG surgery. The differences between CECC and MECC detected in this study remain modest, and a clear advantage regarding the intraoperative microvascular perfusion cannot be claimed for any perfusion system on the basis of the current data from a comparably small cohort. Because the study aimed for comparing the MECC and the CECC as complete systems, it is difficult to attribute the reported difference to a certain component of the circuit. Whether the observed changes can reach clinical significance requires further validation in larger patient cohorts.

\section{References}

1. Newman MF, Kirchner JL, Phillips-Bute B, Gaver V, Grocott H, Jones RH, et al. Longitudinal assessment of neurocognitive function after coronary-artery bypass surgery. N Engl J Med. 2001;344:395-402.

2. Martin BJ, Buth KJ, Arora RC, Baskett RJ. Delirium: a cause for concern beyond the immediate postoperative period. Ann Thorac Surg. 2012;93:1114-20.

3. Liebold A, Khosravi A, Westphal B, Skrabal C, Choi YH, Stamm C, et al. Effect of closed minimized cardiopulmonary bypass on cerebral tissue oxygenation and microembolization. J Thorac Cardiovasc Surg. 2006;131:268-76.

4. Brown WR, Moody DM, Challa VR, Stump DA, Hammon JW. Longer duration of cardiopulmonary bypass is associated with greater numbers of cerebral microemboli. Stroke. 2000;31:707-13.

5. Cryer HG. Therapeutic approaches for clinical ischemia and reperfusion injury. Shock. 1997;8:26-32.

6. Korthuis RJ, Anderson DC, Granger DN. Role of neutrophil-endothelial cell adhesion in inflammatory disorders. J Crit Care. 1994;9:47-71.

7. Wiesenack C, Liebold A, Philipp A, Ritzka M, Koppenberg J, Birnbaum DE, et al. Four years' experience with a miniaturized extracorporeal circulation system and its influence on clinical outcome. Artif Organs. 2004;28:1082-8.

8. Puehler T, Haneya A, Philipp A, Zausig YA, Kobuch R, Diez C, et al. Minimized extracorporeal circulation system in coronary artery bypass surgery: a 10-year single-center experience with 2243 patients. Eur J Cardiothorac Surg. 2011; 39:459-64.

9. Panday GF, Fischer S, Bauer A, Metz D, Schubel J, El Shouki N, et al. Minimal extracorporeal circulation and off-pump compared to conventional cardiopulmonary bypass in coronary surgery. Interact Cardiovasc Thorac Surg. 2009;9:832-6.

10. De Backer D, Ospina-Tascon G, Salgado D, Favory R, Creteur J, Vincent JL. Monitoring the microcirculation in the critically ill patient: current methods and future approaches. Intensive Care Med. 2010;36:1813-25.

11. Weil MH, Nakagawa Y, Tang W, Sato Y, Ercoli F, Finegan R, et al. Sublingual capnometry: a new noninvasive measurement for diagnosis and quantitation of severity of circulatory shock. Crit Care Med. 1999;27:1225-9.

12. Creteur J, De Backer D, Sakr Y, Koch M, Vincent JL. Sublingual capnometry tracks microcirculatory changes in septic patients. Intensive Care Med. 2006;32:516-23.

13. Groner W, Winkelman JW, Harris AG, Ince C, Bouma GJ, Messmer K, et al. Orthogonal polarization spectral imaging: a new method for study of the microcirculation. Nat Med. 1999;5:1209-12.

14. Verdant C, De Backer D. How monitoring of the microcirculation may help us at the bedside. Curr Opin Crit Care. 2005;11:240-4.

15. Zangrillo A, Garozzo FA, Biondi-Zoccai G, Pappalardo F, Monaco F, Crivellari M, et al. Miniaturized cardiopulmonary bypass improves short-term outcome in cardiac surgery: a meta-analysis of randomized controlled studies. J Thorac Cardiovasc Surg. 2010;139:1162-9.

16. Skrabal CA, Steinhoff G, Liebold A. Minimizing cardiopulmonary bypass attenuates myocardial damage after cardiac surgery. ASAIO J. 2007;53:32-5.

17. Anastasiadis $\mathrm{K}$, Argiriadou $\mathrm{H}$, Kosmidis $\mathrm{MH}$, Megari $\mathrm{K}$, Antonitsis $\mathrm{P}$, Thomaidou E, et al. Neurocognitive outcome after coronary artery bypass surgery using minimal versus conventional extracorporeal circulation: a randomised controlled pilot study. Heart. 2011;97:1082-8.

18. Mazzei V, Nasso G, Salamone G, Castorino F, Tommasini A, Anselmi A. Prospective randomized comparison of coronary bypass grafting with minimal extracorporeal circulation system (MECC) versus off-pump coronary surgery. Circulation. 2007;116:1761-7.

19. Bauer A, Kofler S, Thiel M, Eifert S, Christ F. Monitoring of the sublingual microcirculation in cardiac surgery using orthogonal polarization spectral imaging: preliminary results. Anesthesiology. 2007;107:939-45.

20. Harris AG, Sinitsina I, Messmer K. Validation of OPS imaging for microvascular measurements during isovolumic hemodilution and low hematocrits. Am J Physiol Heart Circ Physiol. 2002;282:H1502-9.

21. Halstead JC, Wurm M, Meier DM, Zhang N, Spielvogel D, Weisz D, et al. Avoidance of hemodilution during selective cerebral perfusion enhances neurobehavioral outcome in a survival porcine model. Eur J Cardiothorac Surg. 2007;32: 514-20.

22. Nollert G, Mohnle P, Tassani-Prell P, Reichart B. Determinants of cerebral oxygenation during cardiac surgery. Circulation. 1995;92(9 Suppl):II327-33.

23. Atasever B, Boer C, Goedhart P, Biervliet J, Seyffert J, Speekenbrink R, et al Distinct alterations in sublingual microcirculatory blood flow and hemoglobin oxygenation in on-pump and off-pump coronary artery bypass graft surgery. $J$ Cardiothorac Vasc Anesth. 2011;25:784-90.

24. Skrabal CA, Choi YH, Kaminski A, Steiner M, Kundt G, Steinhoff G, et al. Circulating endothelial cells demonstrate an attenuation of endothelial damage by minimizing the extracorporeal circulation. J Thorac Cardiovasc Surg. 2006; 132:291-6.

25. Wagerle LC, Russo P, Dahdah NS, Kapadia N, Davis DA. Endothelial dysfunction in cerebral microcirculation during hypothermic cardiopulmonary bypass in newborn lambs. J Thorac Cardiovasc Surg. 1998;115:1047-54.

26. Perthel M, Kseibi S, Sagebiel F, Alken A, Laas J. Comparison of conventional extracorporeal circulation and minimal extracorporeal circulation with respect to microbubbles and microembolic signals. Perfusion. 2005;20:329-33.

27. Kamler M, Goedeke J, Pizanis N, Milekhin V, Schade FU, Jakob H. In vivo effects of hypothermia on the microcirculation during extracorporeal circulation. Eur J Cardiothorac Surg. 2005;28:259-65.

28. Thoren A, Nygren A, Houltz E, Ricksten SE. Cardiopulmonary bypass in humans-jejunal mucosal perfusion increases in parallel with well-maintained microvascular hematocrit. Acta Anaesthesiol Scand. 2005;49:502-9.

29. Wiessner R, Gierer P, Schaser K, Pertschy A, Vollmar B, Klar E. [Microcirculatory failure of sublingual perfusion in septic-shock patients. Examination by OPS imaging and PiCCO monitoring]. [Article in German]. Zentralbl Chir. 2009; $134: 231-6$ 